\title{
CAUSAL RELATIONSHIP BETWEEN DRIFTING SUBPULSE PARAMETERS AND SOME PULSAR QUANTITIES
}

\author{
Natasha Shapirovskaya \\ Astro Space Center, P. N. Lebedev Physical Institute
}

\begin{abstract}
Correlation analysis of subpulse spacing $P_{2}$, subpulse bands $P_{3}$, subpulse drift rate with some quantities such as pulsar period $P$, profile width $W_{e}$, characteristic age $\tau$ and others indicates some new casual dependencies not previously confirmed. Relations obtained in this paper possibly point out that interaction between pulsar radio emission and the interstellar environment may exert some influence on the drift appearance.
\end{abstract}

\section{Introduction}

The drifting subpulse phenomenon was observed for the first time by Drake and Craft (1968). Numerous attempts have been made to understand the nature of the drifting subpulses that would be important for further development of pulsar theories. The purpose of this paper is to carry out a statistical analysis of parameters of pulsars which show a regular subpulse drift. Drift appearance is called regular if the $P_{2}$ and $P_{3}$ periods of drift may be measured and kept constant during certain intervals of time. $\left(P_{2}\right.$ is the periodic subpulse spacing; the $P_{3}$ period is the spacing between the successive bands). A similar search of $P_{3}$ period correlations was carried out for a sample of 17 pulsars discussed by Wolszczan (1980). Only 8 pulsars from this sample showed regular drift.

\section{Analysis}

Nowadays more pulsars with regular drift have been recognized. I analyzed three samples: " $L$ ", " $S$ " and "P", put together from published data.

The " $L$ " sample included drift parameters of 18 pulsars with regular drift. Drift parameters of this sample were chosen for the general mode of pulsar emission. The " $S$ " sample included 19 pulsars: 18 from the " $L$ " sample plus PSR $0611+22$ whose drift is not particularly stable. However the $P_{3}$ period of this pulsar has a maximum value, and its drift rate has a minimum value as compared with the drift parameters of other pulsars, which permits us to extend correlations on other scales. The "P" sample included 37 values of drift parameters for all the modes of radio emission of the 19 pulsars of "S" sample (cf. table 1).

$P_{2}$ values for most pulsars are constant for different modes, so statistical analyses of $P_{2}$ periods were carried out only for the " $L$ " and " $S$ " samples.
Correlations of $P_{3}$ periods were calculated for all three samples.

At first it was interesting to consider the earlier known strong dependencies between the $P_{3}$ periods of pulsars with their magnetic fields, $(B \propto$ $\left.(P \dot{P})^{1 / 2}\right]$ and characteristic ages $[\tau \propto P / \dot{P}]$ (Wolszczan 1980). In three samples significant correlations were not found (example of figure 1). The formal difference between my results and previous work results from using a different sample population of pulsars. Probably some pulsars belonging to Wolszczan's sample are not associated with the drifting subpulse phenomenon.

For finding the statistically significant dependencies between drift parameters and different quantities of pulsars, I calculated a correlation matrix of logarithmic values of all parameters from table 1. STATGRAFIC's software package was used for the calculations. More significant correlations were found between the $P_{2}$ periods and the profile width, $W_{e}$ (figure 2 ).

The correlation coefficient was $R=0.82$ with a logarithmic regression slope having a range of values $0.63<\alpha<0.80$ for " $L$ " and " $S$ " samples. The known relationship $W_{\mathrm{e}}-P$ had a poorer correlation $(R=0.52)$ for the same samples. The last relationship permits us to find the numbers of subpulses, $n$, inside the emission window. Assuming $n=W_{e} / P_{2}$ we get $n \propto W_{e}^{0.25 \pm 0.1}$, and the number of subpulses is not more than 4 for all pulsars with regular drift.

$P_{3}$ did not show any significant correlations with the internal parameters of any sample, but demonstrated surprise correlations with external pulsar parameters. The $P_{3}$ periods showed dependence on the distance of the galactic plane and the mean value of the magnetic field averaged along the line of sight

$$
P_{3} \propto Z^{-0.4 \pm 0.03}(R M / D M)^{-0.27 \pm 0.12},
$$$$
0.52<R<0.66
$$ 
SEgYESEIOU CI LOG $P$ ? OR LOG $(Y /(Y / \mathrm{dT}))$

L Emale

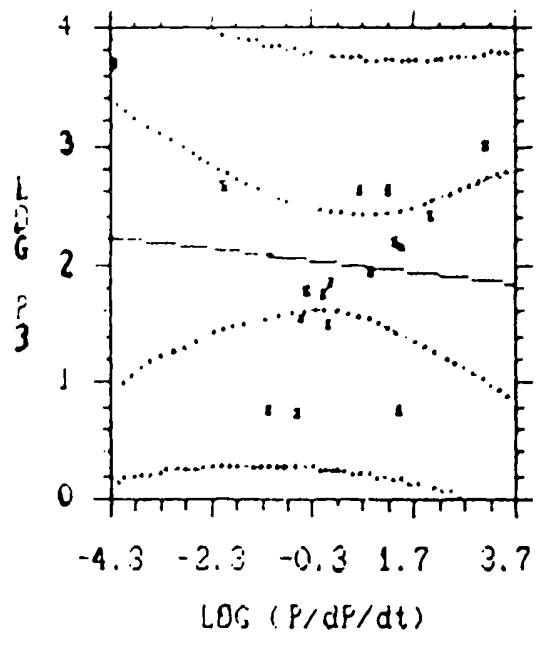

Figure $1 \quad P_{3}$ periods vs. characteristic ages of pulsars.

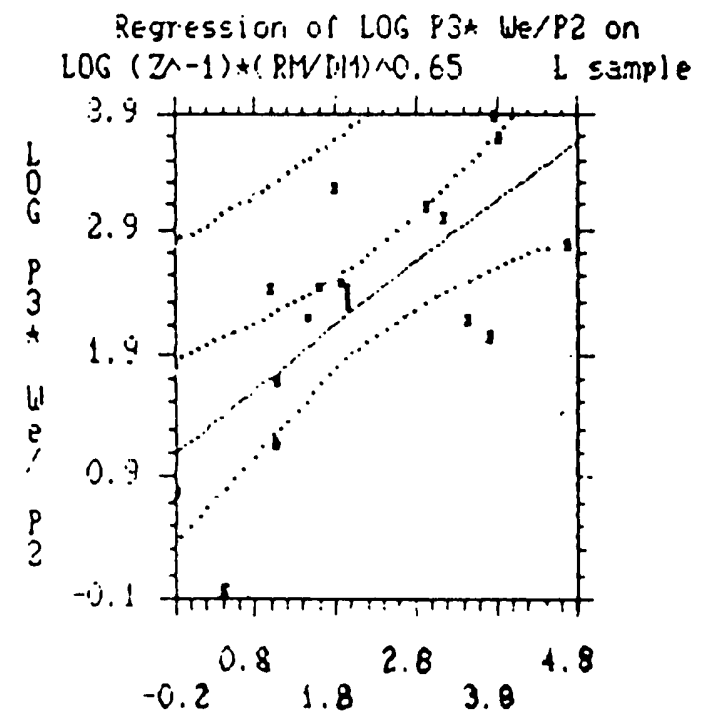

LOCS $(Z n-1) \star((R) \cdot Y D M(n-0) .65)$

Figure 3 Correlations between the actual drift periods and external parameters of pulsars.

It is more interesting to analyze the relationship of real drift periods $\hat{P}_{3}=P_{3} n$. Using $n=W_{\mathrm{e}} / P_{2}$ we get $\hat{P}_{3}=P_{3} W_{\mathrm{e}} / P_{2}$. Results of the correlation analysis for $\hat{P}_{3}$ values confirm the same picture for $P_{3}$ periods but with higher correlation. This was to be expected.

$$
\begin{gathered}
\hat{P}_{3} \propto Z^{-0.5 \pm 0.1}(R M / D M)^{0.5 \pm 0.23}, \\
0.60<R<0.71 .
\end{gathered}
$$

An example of one of the dependencies discussed is given in figure 3 .

As long as the value of $W_{e}$ does not too exartly reflect the real width of the emission window it was interesting to calculate the dependence between $P_{3} / P_{2}$ and the variable $W_{e}, Z$, and $R M / D M$.
Regressicir of LOC P'C IREC On LOS WE IIEËi l sample

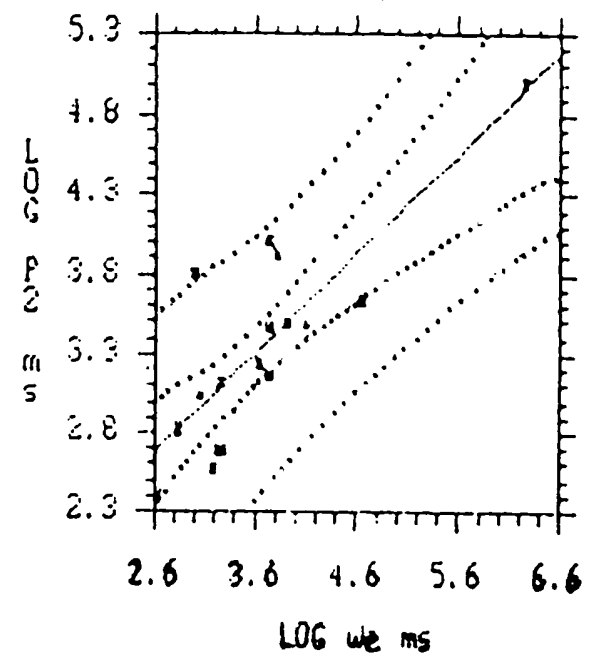

Figure 2 Correlations between the $P_{2}$ periods and profile width $W_{e}$.

Regression of LOS PRrrec/F3 un

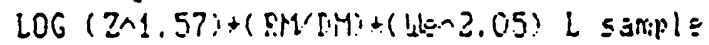

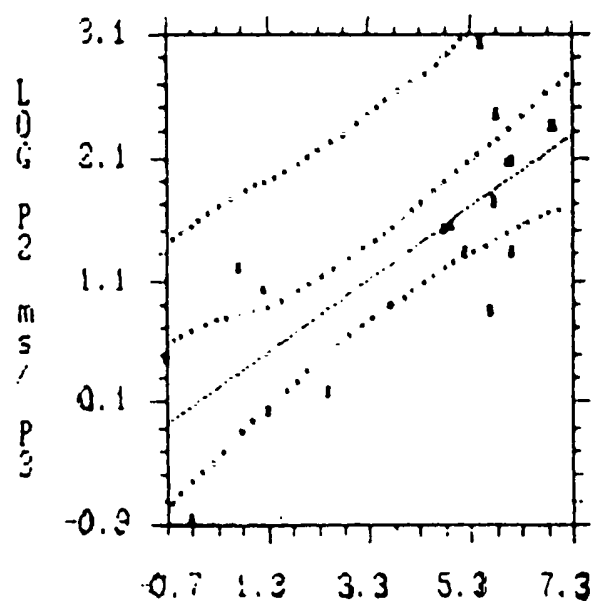

$\operatorname{LOG}(2 \cdot 1.5 T)+(8 \cdot 1 ! \cdot 1, M)+(1,0 \times 2.0 .5)$

Figure 4 Correlations between drift rate and some parameters of pulsars.

Note that the inverse value of $P_{3} / P_{2}$ is determined as a drift rate.

The final statistical analysis of the different pulsar parameters gave the next result (figure 4)

The " $L$ " sample for

$$
P_{2} / P_{3} \propto W_{e}^{0.61} Z^{0.47}(R M / D M)^{0.3},
$$

$$
R=0.77,
$$

$T$ value $=4.78$ and probability level $=2 \times 10^{-4}$.

The "S" sample for

$$
\begin{gathered}
P_{2} / P_{3} \propto W_{\mathrm{e}}(Z R M / D M)^{0.42}, \\
R=0.71,
\end{gathered}
$$

$T$ value $=4.2$ and probability level $=6 \times 10^{-4}$. 
Table 1

\begin{tabular}{|c|c|c|c|c|c|c|c|c|c|c|c|}
\hline $\mathrm{N}$ & PSR & $\begin{array}{c}P \\
(\mathrm{sec})\end{array}$ & $\begin{array}{l}P_{2} \\
\left({ }^{\circ}\right)\end{array}$ & $\begin{array}{r}P_{3} \\
(P) \\
\end{array}$ & $\begin{array}{r}W_{e} \\
(\mathrm{~ms})\end{array}$ & $\begin{array}{r}\dot{P} \\
\left(10^{-15}\right) \mathrm{s} / \mathrm{s} \\
\end{array}$ & $\begin{array}{r}D M \\
\left(\mathrm{pccm}^{-3}\right) \\
\end{array}$ & $\begin{array}{r}R M \\
(\mathrm{rad} / \mathrm{m}) \\
\end{array}$ & $\begin{array}{c}R \\
(\mathrm{kpc})\end{array}$ & $\begin{array}{r}Z \\
(\mathrm{kpc})\end{array}$ & ref \\
\hline 1 & $0031-07$ & 0.943 & 21.0 & 7.0 & 42 & 0.41 & 10.89 & 9.8 & 0.39 & 0.37 & 1 \\
\hline 2 & $0148-06$ & 1.465 & 8.0 & 13.9 & 60 & 0.45 & 25.10 & 2.0 & 0.97 & 0.88 & 2 \\
\hline 3 & $0301+19$ & 1.388 & 6.2 & 6.4 & 42 & 1.30 & 15.69 & 8.3 & 0.56 & 0.31 & 1 \\
\hline 4 & $0320+39$ & 3.032 & 3.8 & 8.5 & 42 & 0.71 & 25.80 & 58.0 & 0.89 & 0.22 & 3 \\
\hline 5 & $0809+71$ & 1.292 & 14.0 & 11.0 & 45 & 0.17 & 5.76 & 11.7 & 0.17 & 0.09 & 1 \\
\hline 6 & $0818-13$ & 1.238 & 3.8 & 4.7 & 24 & 2.11 & 41.00 & 1.2 & 1.50 & 0.32 & 4 \\
\hline 7 & $0826-34$ & 1.849 & 29.0 & 14.0 & 520 & 1.00 & 47.30 & 59.0 & 0.45 & 0.02 & 5 \\
\hline 8 & $0943+10$ & 1.098 & 8.4 & 2.2 & 38 & 3.53 & 15.35 & 13.3 & 0.56 & 0.38 & 6 \\
\hline 9 & $1112+50$ & 1.656 & 3.2 & 6.0 & 27 & 2.49 & 9.16 & 3.2 & 0.32 & 0.28 & 7 \\
\hline 10 & $1632+24$ & 0.491 & 33.1 & 2.2 & 20 & 0.12 & 23.80 & 31.0 & 0.88 & 0.56 & 8 \\
\hline 11 & $1822-09$ & 0.769 & 8.0 & 40.0 & 17 & 52.32 & 19.90 & 65.0 & 0.51 & 0.01 & 9 \\
\hline 12 & $1845-01$ & 0.659 & 12.4 & 14.2 & 26 & 5.20 & 159.10 & 580.0 & 3.70 & 0.003 & 8 \\
\hline 13 & $1918+19$ & 0.821 & 14.5 & 5.8 & 50 & 0.90 & 154.40 & 160.0 & 5.00 & 0.23 & 8 \\
\hline 14 & $1919+21$ & 1.337 & 4.0 & 4.5 & 25 & 1.35 & 12.43 & 16.5 & 0.33 & 0.02 & 1 \\
\hline 15 & $1944+17$ & 0.441 & 17.0 & 20.0 & 21 & 0.02 & 16.30 & 28.0 & 0.43 & 0.03 & 1 \\
\hline 16 & $2016+28$ & 0.558 & 7.0 & 9.0 & 14 & 0.15 & 14.90 & 34.6 & 1.30 & 0.09 & 1 \\
\hline 17 & $2303+30$ & 1.576 & 3.8 & 2.1 & 17 & 2.90 & 49.90 & 84.0 & 1.90 & 0.86 & 1 \\
\hline 18 & $2319+60$ & 2.256 & 6.0 & 8.0 & 105 & 7.04 & 93.80 & 230.0 & 2.80 & 0.03 & 10 \\
\hline 19 & $0611+22$ & 0.335 & 2.0 & 75.0 & 8 & 59.63 & 96.70 & 67.0 & 3.30 & 0.13 & 11 \\
\hline 20 & $1112+50$ & & 1.4 & & & & & & & & \\
\hline 21 & $1112+50$ & & 6.1 & & & & & & & & \\
\hline 22 & $1822-09$ & & 6.0 & 11.0 & & & & & & & \\
\hline 23 & $0031-07$ & & & 13.0 & & & & & & & \\
\hline 24 & $0031-07$ & & & 4.0 & & & & & & & \\
\hline 25 & $0148-06$ & & & 26.7 & & & & & & & \\
\hline 26 & $0943+10$ & & & 2.5 & & & & & & & \\
\hline 27 & $0943+10$ & & & 1.9 & & & & & & & \\
\hline 28 & $0826-34$ & & & 19.0 & & & & & & & \\
\hline 29 & $1918+19$ & & & 3.9 & & & & & & & \\
\hline 30 & $1918+19$ & & & 2.5 & & & & & & & \\
\hline 31 & $2016+28$ & & & 15.0 & & & & & & & \\
\hline 32 & $2016+28$ & & & 3.0 & & & & & & & \\
\hline 33 & $2303+30$ & & & 2.4 & & & & & & & \\
\hline 34 & $2303+30$ & & & 1.8 & & & & & & & \\
\hline 35 & $2319+60$ & & & 4.0 & & & & & & & \\
\hline 36 & $2319+60$ & & & 3.0 & & & & & & & \\
\hline 37 & $0611+22$ & & & 100.0 & & & & & & & \\
\hline
\end{tabular}

Title of column: 1-pulsar number; 2-pulsar name; 3-basic period; 4- $P_{2}$ period; $5-P_{3}$ period; 6- $W_{e}$, equivalent width, the full width at half intensity; $7-d P / d t$, period derivative; $8-D M$, dispersion measure; $9-R M$, rotation measure; $10-R$, distance to pulsars; $11-Z$, height above the galactic plane; 12 -references: 1 ) Manchester and Taylor 1977; 2) Biggs et al. 1985; 3) Izvekova et al. 1982; 4) Biggs et al. 1987; 5) Biggs et al. 1985a; 6) Sieber and Oster 1975; 7) Wright, Sieber and Wolszczan 1986; 8) Hankins and Wolszczan 1987; 9) Fowler et al. 1980; 10) Wright and Fowler 1981 ; 11) Ferguson and Boriakoff 1980.

The "P" sample for

$$
\begin{gathered}
P_{2} / P_{3} \propto W_{\mathrm{e}}^{1.2}(Z R M / D M)^{0.60}, \\
R=0.74,
\end{gathered}
$$

$T$ value $=6.52$ and probability level $=2 \times 10^{-7}$.
The relations obtained in this paper are unusual and it is difficult to explain in the frames of known theoretical models. It is possible that the interaction of the pulsar radio beam with the interstellar medium exerts some influence on the drift parameters. 